\title{
Cardiovascular Adaptation in Athletes
}

\author{
Richard Kovacs, $\mathrm{MD}^{1}$ \& Aaron L. Baggish, MD $^{2}$ \\ ${ }^{1}$ Krannert Institute of Cardiology, Indiana University School of Medicine, Indianapolis, IN \\ ${ }^{2}$ Cardiovascular Performance Center, Massachusetts General Hospital, Boston, MA
}

Word Count: 5,690

Key Words: athlete's heart, sports cardiology, exercise, athlete

Corresponding Author Contact Information:

Richard Kovacs, MD

Supported in part by the Indiana University Health / Indiana University School of Medicine Strategic Research Initiative (RJK).

This is the author's manuscript of the article published in final edited form as:

Kovacs, R., \& Baggish, A. L. (2016). Cardiovascular adaptation in athletes. Trends in Cardiovascular Medicine, 26(1), 46-52. http://doi.org/10.1016/j.tcm.2015.04.003 


\section{Magnitude and Complexity of the Athlete Population}

A recent state of the art paper ${ }^{1}$ summarized the role of cardiovascular specialty in respect to an explosion in the number of athletes training for and participating in organized and recreational sports. More than 44 million boys and girls participate in youth sports each year, nearly 10 million at the high school level, and nearly half a million at the collegiate level. 541,000 runners finished a marathon in 2013 in the U.S. Professional sports teams employ thousands of athletes. The number of older athletes ( $>35$ years) is increasing, but difficult to estimate. Many of these athletes have pre-existing heart disease, including the adult survivors of congenital heart disease, whose numbers now exceed the number of children with congenital heart disease.

The variety of sports has also dramatically increased, with athletes participating in traditional sports such as football, basketball and track, but also emerging and extreme sports that push the limits of human endurance. 28 sports will be contested at the 2016 Summer Olympics, and many of those sports are subdivided into disciplines that each has unique demands on the cardiovascular system.

The ethnic diversity of the athlete population is increasing, but also may be increasingly sport-specific. African-Americans comprised $76 \%$ of all NBA players, but only $8 \%$ of major league baseball players. Statistics on youth and recreational sports are more difficult to come by, but can be expected to reflect the increasing diversity in the population in general.

These factors are all important to consider in framing the discussion of cardiovascular adaptation in the athlete, as adaptation will be dependent on both the characteristics of the sport and the participant. One size will not fit all, and the lessons learned in one sport may not translate to the athletes in another sport. The field has developed to the point where experts have called for new paradigms in the care of this unique population by the cardiovascular care team. ${ }^{1}$

\section{Evolving Tools to Characterize Cardiovascular Adaptation in Athletes}

Cardiovascular adaptation in the athlete was limited to description of physical findings until the advent of modern imaging techniques. Morganroth and colleagues ${ }^{2}$ described the assessment of cardiac adaptation through the use of modern imaging techniques, such as echocardiography for the assessment of chamber size and wall thickness. ${ }^{2}$ In 2014, multimodality imaging is readily available to assess cardiac structure and function in the athlete, with cardiac CT, cardiac MR, and advanced echo techniques, such as strain imaging or 3-D available to define cardiac anatomy.

Cardiac electrophysiology was limited in the past to evaluation by resting electrocardiogram or hard wired stress tests. Ambulatory monitoring with standard Holter recorders or event monitors remains difficult in any person during exercise and nearly impossible during competition. In 2014, a variety of wearable sensors, miniaturized event recorders and even implantable devices allow for easier assessment of electrophysiological changes in athletes.

Classification of sports into categories with expected adaptive changes has also evolved. These categories and have been incorporated in guidelines for athletic participation. ${ }^{3}$ Sports-specific databases are being developed, with the National Football League and the National Basketball Association performing 
screening imaging exams, and recording data to allow for the establishment of normative values in professional sports. Collegiate programs have been developed to evaluate incoming student-athletes, and have demonstrated the rapid pace of cardiac adaptation that occurs upon beginning collegiate training programs.

\section{The Need to Differentiate Normal Adaptation from Disease}

Increasing numbers of competitive and highly trained athletes are presenting to both primary care providers and specialists for initial clearance to play as well as for return to play decisions after potential cardiac symptoms or a suspicious event. These decisions have important ramifications for the athlete, their family, team, league and community. Athletes want to return to play, whether for the personal reasons of an amateur or the financial incentives of a professional. Accurate and timely decisions need to be made to avoid interruptions in training or competition. Several states have enacted laws that regulate return to play in student athletes. All parties want safety for the athlete. The medical provider faces the special issues of medical liability.

The overlap between physiology and pathology is important in clinical medicine, but is even more crucial in the athlete population, where several important causes of athletic sudden cardiac death can be difficult to differentiate from adaptation. We refer specifically (but not exclusively) to the left ventricular hypertrophy of training, as opposed to the pathologic hypertrophy of a hypertrophic cardiomyopathy, right ventricular chamber enlargement as opposed to arrhythmogenic right ventricular cardiomyopathy, or prolonged ventricular repolarization due to a training-related bradycardia as opposed to a congenital long QT syndrome.

The cardiovascular specialist, as well as the primary care provider involved in screening athletes or determining return to play needs to understand cardiac adaptation to athletic training. In addition, the practitioner should also be aware of the particular demands of the sport, understand what is expected for the age and level of training of the individual athlete they are faced with, and be aware of confounding factors, such as performance enhancing drugs, that might interfere with the ability to differentiate adaptation from disease.

\section{Physiologic demands of sports}

Basics of Exercise Physiology. The physiology underlying competitive athletics and vigorous exercise is complex. Physical activity requires the coordinated and purposeful contraction of specific groups of skeletal muscle that are recruited by the central nervous system to perform specific tasks. The actions that are inherent in sporting activity, referred to in aggregate as "external work", include but are not limited to actions such as running, pedaling, and jumping. These actions can be quantified precisely using sport-specific metrics including running speed or cycling wattage. For any given athletes, there is a direct correlation between the magnitude of external work and the amount of internal work required to complete any specific task. Internal work, the total metabolic cost of physical activity, is a broad term that encompasses substrate utilization, substrate transport, and metabolic byproduct removal and is most commonly quantified by measurement of oxygen consumption $\left(\mathrm{VO}_{2}\right)$. The cardiovascular system plays a key role in dynamic process of internal work. Specifically, the primary goal the cardiovascular system is to simultaneously provide the activated skeletal muscle with energy rich substrate (i.e. glucose, fatty acids, and oxygen) and to return the 
byproducts of metabolism to the organs responsible for their disposal. This process is accomplished by increases in cardiac output, the magnitude of which is tightly coupled to the needs of the activated skeletal muscle groups. Fundamental mechanisms responsible for increased cardiac output in the context of exercise include increases in heart rate, ventricular stroke volume, and peripheral arterial vasodilation.

The Physiology of Cardiac Remodeling. (Figure 1) Exercise-induced cardiac remodeling (EICR) is stimulated by the pressure and volume stressors that accompany increases in both external and internal work. ${ }^{4}$ The specific stimuli inherent in the exercise response can be dichotomous into isometric and isotonic physiology. Isotonic stress refers to the movement of large quantities of blood through the cardiovascular system. Endurance sporting disciplines including long-distance running, Nordic skiing, rowing, and cycling are characterized by a predominance of isotonic stress. From the perspective of the heart, isotonic physiology imparts a "volume" load which is felt by all 4 chambers and accompanying great vessels. As discussed in detail below, isotonic physiology, when applied repetitively over a sustained period of time, typically produces chamber dilation. In contrast, isometric stress refers to the generation of high intravascular pressure. Sporting disciplines that require short but intense, repetitive bursts of activity such as power weight lifting, American-style football line position play, and martial arts produce the most robust isometric physiology. The coordinated and often near maximal contraction of large skeletal muscle groups coupled with surges in catecholamine release cause transient increases in systemic blood pressure in excess of $400 \mathrm{mmHg}{ }^{5,6}$ From the perspective of the heart, isometric physiology imparts a "pressure" load that is focused largely on the left ventricle due to the fact that a competent mitral valve spares the remainder of a central cardiac apparatus. It has been shown that Valsalva physiology during isometric activity may reduce or negate the magnitude of left ventricular pressure overload due to the fact that simultaneous increases in intrathoracic pressure and intracardiac pressure effectively normalize transmural pressure, the principal determinant of myocardial work. ${ }^{7}$ However, most athletic activities characterized by isometric physiology are done without the prolonged breath-holding required to neutralize transmural left ventricular pressure.

Classification of Sports. Recognition of these two principal forms of cardiovascular stress serves as the basis for sport classification as a function of physiology. The 36th Bethesda Conference and its up-coming revision propose a classification of sports based on the matrix integrating the relative intensities of isotonic and isometric activity. ${ }^{3}$ Sports characterized by relatively pure isotonic stress include long-distance running, soccer, and cross-country skiing. Relatively pure isometric stress sports include weightlifting, martial arts, and track and field throwing events. Athletes participating in these "pure stress" sporting disciplines have been used to define the concept of sports specific exercise-induced cardiac remodeling. Isotonic stress stimulates a form of exercise-induced remodeling characterized by biventricular dilation, biatrial dilation, and enhanced left ventricular diastolic function. In contrast, isometric stress stimulates remodeling confined to the left ventricle that is typically characterized by mild degrees of concentric left ventricular hypertrophy with unchanged or relative impairment of diastolic function. It is noteworthy that many of the most popular sports involve significant amounts of both isotonic and isometric cardiovascular stress. As anticipated, individuals that participate in "physiology overlap sports including" (i.e. concomitant high isometric/high isotonic) including competitive cycling and rowing typically demonstrate the most robust cardiac adaptations with elements of both pressure and volume mediated remodeling. 


\section{Myocardial adaptations to exercise}

Left Heart. Left ventricular remodeling in response to exercise training has been the topic of extensive study. Early cross-sectional data from large heterogeneous cohorts of European athletes demonstrated a high prevalence of left ventricular cavity enlargement and increased wall thickness. ${ }^{8}$ Depending upon the choice of cut point, as many as $30 \%$ of healthy competitive athlete can be expected to demonstrate LV chamber dimensions that exceed the upper limits of normal. In similar fashion, mild increases in LV wall thickness, typically ranging from 12-14 mm, are commonly observed among trained athletes. ${ }^{9}$ LV geometry among athletes is sport specific. ${ }^{10-12}$ Individuals who participate in sports with significant isotonic physiology tend to develop eccentric left ventricular remodeling which is characterized by balanced chamber dilation and wall thickening. In contrast, athletes participating in sports with predominant isometric physiology most commonly develop concentrate left ventricular remodeling which is characterized by wall thickening in the absence of chamber dilation.

Functional adaptations of the LV to exercise training appear to be similarly sports specific. The majority of published data suggest that isotonic training leads to enhanced diastolic function as measured both by Doppler echocardiography and invasive hemodynamics. In contrast, athletes participating in isometric sports typically demonstrate normal or slightly reduced left ventricular diastolic function. In aggregate, neither form of exercise training has a substantial effect on resting left ventricular systolic function. Most data demonstrate that left ventricular function is almost always within the normal range as measured both by ejection fraction and more advanced noninvasive indices of systolic function. ${ }^{13}$ However, in clinical practice it is not unusual to see low normal or slightly depressed resting left ventricular function among trained endurance athletes. ${ }^{14}$ In our experience; this represents physiologic reserve rather than subclinical pathology as evidenced by normal augmentation and supernormal exercise capacity during provocative laboratorybased exercise testing. Among athletes who participate in mixed physiology sports (i.e. team sport athletes) LV geometry and function are variable. Most often, the physiology required to participate a specific field position dictates the form of remodeling observed. ${ }^{15}$

Right Heart. Recently, a growing number of investigators have focused on the right ventricular responses to exercise training. Isotonic physiology imparts a balanced biventricular stress and therefore often results in right ventricular dilation. Several recent cardiac imaging studies using both echocardiography and magnetic resonance imaging document a high prevalence of right ventricular dilation among endurance trained athletes. ${ }^{16,17}$ Not unlike the left ventricle, resting RV systolic function may be mildly reduced among trained endurance athletes and likely reflects the substantial contractile reserve afforded by physiologic RV dilation. ${ }^{18}$ Given the difficulty of quantifying right ventricular hypertrophy by noninvasive imaging, there are limited data addressing whether the RV thickens in response to exercise. The available literature would suggest that right ventricular hypertrophy is an unusual response to exercise training in the absence of some form of concomitant pulmonary hypertension.

The right ventricle appears largely unresponsive to isometric sports training. ${ }^{19}$ This observation is best explained by the fact that the pulmonary vasculature and right heart are typically shielded from the intense 
intravascular pressures inherent in isometric activity by the mitral valve. Therefore, right ventricular dilation and/or hypertrophy among strengthening athletes should be considered suspicious for underlying pathology rather than adaptive physiology.

Temporal Determinants of Cardiac Adaptation. EICR requires repetitive and sustained exposure to an exercise stimulus. At the present time, the minimally required intensity and duration of exercise required to stimulate this process remains largely unexplored. This knowledge gap is due to the fact that the vast majority of the above described attributes of the "athlete's heart" has been defined using cross-sectional sectional study designs. Cross-sectional studies utilize measurements of cardiac structure and/or function that are made in a specific athlete population a single time point. This approach is popular due to the fact that it can be completed with relative logistic ease and minimal resource investment. However, crosssectional study designs do not permit investigators to establish any cause-and-effect relationships about the data they generate, nor do they facilitate the ability to examine the dose response and temporal relationships between exercise and cardiac remodeling. Several longitudinal studies of EICR have recently been completed and have established with certainty, a mechanistic link between exercise exposure and sport/physiology-specific EICR. ${ }^{11,20,21}$ To date, the few longitudinal studies that have been completed and published have involved relatively short duration exercise exposure and have focused on relatively young athletes. Work from the Harvard Athlete Initiative has shown that 90 days of intense team-based training is sufficient to produce the sport-specific forms of left ventricular remodeling described above. One prior study of Tour de France cyclists demonstrated progressive LV chamber dilation with concomitant reduction in LV wall thickness over a three-year period of professional cycling. ${ }^{22}$ Longer term repeated measure studies that account for key confounding variables including athlete age, ethnicity, gender, and exercise dose/physiology will be required to define the relationships between exercise exposure (both intensity and duration) and cardiac remodeling.

Regression with Exercise Detraining. Cardiovascular adaptations to exercise may regress following exercise abstinence or reduction. The sentinel report documenting regression of cardiac adaptation in the setting of physical deconditioning was published in 1993. ${ }^{23}$ Maron et al studied 6 elite athletes (participants in the 1988 Seoul Olympic Games) with marked left ventricular hypertrophy (septal wall thickness $=13.8 \pm 0.9 \mathrm{~mm}$ ) after variable periods (6-34 weeks) of voluntary training reduction. Following detraining, this small cohort of athletes demonstrated significant regression of left ventricular hypertrophy (septal wall thickness $=10.5 \pm 0.5$ $\mathrm{mm}$ ). This important study lead to the concept of using prescribed deconditioning to differentiate marked physiologic hypertrophy from mild pathologic cardiomyopathy. A decade later, Pelliccia et al examined 40 elite male athletes with eccentric left ventricular hypertrophy (LV chamber diameter $=61.2 \mathrm{~mm}$, LV wall thickness $=12.0 \pm 1.3 \mathrm{~mm}$, LV mass $=194 \mathrm{~g} / \mathrm{m}$ ) after more extensive detraining $(5.6 \pm 3.8$ years $) .{ }^{24} \mathrm{In}$ this setting, both left ventricular mass $(140 \pm 21 \mathrm{~g} / \mathrm{m})$ and wall thickness $(10.1 \pm 0.8 \mathrm{~mm})$ returned to normal values in all athletes while persistent left ventricular cavity dilation remained in $22 \%$ of the cohort. Although further confirmation is required, this study suggests that some aspects of exercise-induced cardiac remodeling (i.e. chamber dilation) may be permanent or at least persistent for long periods following exercise abstinence.

To our knowledge, the response of concentric left ventricular hypertrophy, the geometric pattern associated with strength-based training, to exercise abstinence has been examined in only one small cohort. ${ }^{25}$ Weiner 
and colleagues studied 5 collegiate male football athletes with marked concentric left ventricular hypertrophy (LV mass $=139 \pm 7 \mathrm{~g} / \mathrm{m}^{2}$, LV wall thickness $=12.4 \pm 0.5 \mathrm{~mm}$ ) before and after 6 months of prescribed deconditioning. In the study, statistically significant reductions in left ventricular mass, left ventricular wall thickness, and left atrial size, amounting to normalization of parameters, was observed among all participants. Despite these important prior studies, there is much to be learned about how the exercise adapted cardiovascular system responds to training cessation. Larger studies of healthy athletes with standardized detraining protocols coupled with longitudinal studies examining cardiac morphology among athletes with recently diagnosed cardiomyopathy conditions necessitating sport restriction are needed. An important objective of future work will be to clarify the absolute magnitude and temporal nature of regression to ensure that clinicians are able to differentiate true physiologic regression from simple regression toward the population mean. Such efforts are necessary to clarify the physiology of cardiac regression and the role of prescribed detraining for the purpose of the differentiating physiology from disease.

Electrophysiological Adaptation. Athletic training induces changes in cardiac electrophysiology both directly at the organ level, by chamber enlargement and hypertrophy, as well as indirectly, through changes in autonomic input to the heart. The standard 12 lead electrocardiogram has been extensively used in evaluation of athletes at all levels of competition, and information is also available from continuous ECG monitoring, measures of heart rate variability, vector loops and signal averaged ECGs.

The ECG correlates of structural adaptation of the heart, i.e. left ventricular hypertrophy, right ventricular hypertrophy, left and right atrial enlargement and incomplete right bundle branch block are well described and expected. Racial and ethnic differences in ECG patterns have been identified, especially in patterns of repolarization. To differentiate from pathologic processes, such as inherited hypertrophic cardiomyopathies or arrhythmogenic right ventricular cardiomyopathy, ECG scoring systems have been developed by consensus to assist clinicians in differentiating normal adaptation from cardiac disease. ${ }^{26-28}$

Athletic training, especially endurance training, also has profound effects on the autonomic nervous system, and these effects are manifest in alterations of cardiac electrophysiology. The primary autonomic alteration is an increase in parasympathetic tone, with resultant sinus bradycardia, sinus arrhythmia, first degree atrioventricular block and Mobitz I second degree atrioventricular block all common findings in the athlete.

The current ECG scoring systems recognize the profound bradycardia produced by training, and consider sinus rates greater than 30 beats per minute on resting 12 lead ECG to fall within the normal range for the athlete. During long term recordings, increased heart rate variability with increased spectral power in the high frequency range is detected, suggesting the heightened vagal tone. ${ }^{29}$

Heightened parasympathetic tone and resultant resting sinus bradycardia in the athlete may have indirect effects on cardiac electrophysiology. Change in the refractory period of an accessory pathway has been postulated as a mechanism that might "unmask" Wolff-Parkinson-White patterns in athletes. ${ }^{30}$ Resting bradycardia also influences cardiac ventricular repolarization, as measured in the QT interval of the ECG. QT prolongation is expected with slower resting heart rates, and differentiation of normal prolongation from pathologic prolongation due to inherited channelopathies can be problematic. ${ }^{31}$ QT correction factors are not perfect at compensating for the dependence of QT on heart rate, especially the commonly used Bazett 
correction factor. ${ }^{32}$

Autonomic modulation of cardiac ventricular electrophysiology is also important in the athlete. Athletes exhibit an increased incidence of early repolarization pattern on 12 lead ECG, a pattern that can evolve with intense training and dissipate with detraining. ${ }^{33}$ The mechanism is uncertain, but is postulated to be due to parasympathetic modulation of ventricular repolarization, increasing regional dispersion of repolarization of the ventricular myocardium ${ }^{37}$. This may be a direct effect of the vagus nerve in the ventricle, but could also be due to heightened vagal effects on sympathetic nervous activity - termed "accentuated antagonism" and first described at the cellular level by Bailey and Watanabe. ${ }^{34}$ This same alteration of ventricular repolarization by heightened vagal tone may also be the mechanism of increased T wave amplitude in the athlete heart. $^{35}$

The description of an association between early repolarization patterns and an increased risk of sudden cardiac death ${ }^{40}$ makes differentiation of exercise-induced early repolarization from the variant associated with sudden death important to the clinician. The most effective way to differentiate may be to recognize that while early repolarization in the young athlete appears dynamically at times of peak fitness, the increased mortality associated with patterns of early repolarization appears to be confined to the fourth and fifth decades of life.

\section{Performance Enhancing Drugs (PEDs)}

The use of PEDs has increased in popularity over the last several decades among elite and recreational athletes. ${ }^{36}$ Definitive studies documenting the impact of PEDs on cardiac structure and function are lacking. Several small cross-sectional observational efforts have examined the impact of anabolic androgenic steroids on cardiac structure and function. ${ }^{37-39}$ In aggregate, these studies suggest that anabolic androgenic steroids may facilitate mild left ventricular hypertrophy with associated reductions in left ventricular diastolic and systolic function. At present, the dose-response relationship between steroid use and left ventricular morphology/dysfunction remains unknown. Further, the implications of the observed impairments of left ventricular function among steroid users will remain speculative pending future work that involves firm clinical end points.

\section{Clinical Approach to Athlete with "Gray-Zone" Cardiac Morphology}

There can be substantial quantitative overlap between marked physiologic adaptation and mild cardiomyopathic disease. Historically, the "gray-zone" athlete was a term used to describe the individual who presented with LV wall thicknesses that exceeded normal values thus raising concern for pathologic hypertrophic cardiomyopathy. In contemporary sports cardiology practice, gray zone athletes come in numerous forms including those with RV dilation (concerning for ARVC), LV dilation (concerning for dilated cardiomyopathy), and hypertrabeculation (concerning for non-compaction). The diagnostic approach to such athletes is often complex and a while a detailed discussion of this clinical challenge is beyond the scope of this review, several general principals merit consideration. The likelihood that an abnormal cardiac measurement in an athlete represents true disease versus benign adaptation begins with consideration of pre-test probability. The pre-test probability of underlying disease is higher in symptomatic athletes and those with a family history of inherited cardiac disease or unexplained death/syncope than in an asymptomatic athlete deemed to be abnormal during pre-participation screening. While we encourage the 
practicing clinician to keep this concept in mind at all times, occult disease in asymptomatic athletes does exist and almost certainly comes with future risk of adverse outcomes during vigorous physical activity. As such, thoughtful assessment, almost always relying complementary diagnostic testing, is required in most cases. ${ }^{40}$ Echocardiography remains the initial test of choice among athletes with suspected myocardial pathology. Recent advances in functional echocardiography including the use of tissue Doppler imaging and perhaps speckle-tracking strain imaging provide important complements to the more rudimentary techniques of 2D-gray scale and conventional Doppler imaging. The advent and widespread dissemination of both cardiac magnetic resonance imaging (CMRI) and cardiac computed tomography (cCT) have proven useful in this clinical context. We routinely use $\mathrm{CMRI}$ as a follow-up to echocardiography to characterize left and right ventricular architecture in gray zone athletes and utilize $\mathrm{CCT}$ to evaluate for coronary origins when any ambiguity persists following echocardiography. It should be emphasized that evaluation of the gray zone athlete extends beyond imaging and typically requires ambulatory rhythm monitoring, provocative exercise testing, and in some cases prescribed detraining. When a diagnosis of cardiac disease has been firmly established, we use the framework provided by current consensus documents to facilitate a patient-centered discussion regarding the risks and benefits of continued athletic participation. ${ }^{41}$ Among athletes who are removed from sport or who chose to abstain from competitive athletics, we work to encourage the more moderate forms of exercise that are associated with optimal health and longevity. ${ }^{42}$

\section{Conclusions}

Increasing numbers of athletes are training and competing in an expanding variety of strength and endurance sports. Cardiovascular adaptation to training is sport-specific and time dependent. Structural changes to the heart occur in response to pressure and/or volume loading during training and competition.

Electrophysiological changes occur in parallel o to the structural changes, either as a consequence of chamber and/or hypertrophy, but also as a consequence of altered autonomic tone. The clinician caring for athletes needs fundamental understanding of the normal cardiovascular adaptation to training, and how this differs from pathology. Sports cardiology is a growing field of cardiovascular experts who deal with the complex interaction of athletic activity and the heart. 


\section{REFERENCES}

1. Lawless CE, Olshansky B, Washington RL, Baggish AL, Daniels CJ, Lawrence SM, Sullivan RM, Kovacs RJ and Bove AA. Sports and exercise cardiology in the United States: cardiovascular specialists as members of the athlete healthcare team. Journal of the American College of Cardiology. 2014;63:1461-72.

2. Morganroth J, Maron BJ, Henry WL and Epstein SE. Comparative left ventricular dimensions in trained athletes. Annals of internal medicine. 1975;82:521-4.

3. Mitchell JH, Haskell W, Snell P and Van Camp SP. Task Force 8: classification of sports. Journal of the American College of Cardiology. 2005;45:1364-7.

4. Baggish AL and Wood MJ. Athlete's heart and cardiovascular care of the athlete: scientific and clinical update. Circulation. 2011;123:2723-35.

5. MacDougall JD, Tuxen D, Sale DG, Moroz JR and Sutton JR. Arterial blood pressure response to heavy resistance exercise. J Appl Physiol. 1985;58:785-90.

6. Palatini P, Mos L, Munari L, Valle F, Del Torre M, Rossi A, Varotto L, Macor F, Martina S, Pessina AC and et al. Blood pressure changes during heavy-resistance exercise. Journal of hypertension Supplement : official journal of the International Society of Hypertension. 1989;7:S72-3.

7. Haykowsky M, Taylor D, Teo K, Quinney A and Humen D. Left ventricular wall stress during leg-press exercise performed with a brief Valsalva maneuver. Chest. 2001;119:150-4. 8. Pelliccia A, Culasso F, Di Paolo FM and Maron BJ. Physiologic left ventricular cavity dilatation in elite athletes. Annals of internal medicine. 1999;130:23-31.

9. Sharma S, Maron BJ, Whyte G, Firoozi S, Elliott PM and McKenna WJ. Physiologic limits of left ventricular hypertrophy in elite junior athletes: relevance to differential diagnosis of athlete's heart and hypertrophic cardiomyopathy. Journal of the American College of Cardiology. 2002;40:1431-6.

10. D'Andrea A, Limongelli G, Caso P, Sarubbi B, Della Pietra A, Brancaccio P, Cice G, Scherillo M, Limongelli F and Calabro R. Association between left ventricular structure and cardiac performance during effort in two morphological forms of athlete's heart. International journal of cardiology. 2002;86:177-84.

11. Baggish AL, Wang F, Weiner RB, Elinoff JM, Tournoux F, Boland A, Picard MH, Hutter AM, Jr. and Wood MJ. Training-specific changes in cardiac structure and function: a prospective and longitudinal assessment of competitive athletes. J Appl Physiol. 2008;104:1121-8.

12. Pluim BM, Zwinderman AH, van der Laarse A and van der Wall EE. The athlete's heart. A meta-analysis of cardiac structure and function. Circulation. 2000;101:336-44.

13. Baggish AL, Yared KL, Wang F, Weiner RB, Hutter Jr AM, Picard MH and Wood MJ. The Impact of Endurance Exercise Training on Left Ventricular Systolic Mechanics. American journal of physiology. 2008.

14. Baggish AL, Yared K, Weiner RB, Wang F, Demes R, Picard MH, Hagerman F and Wood MJ. Differences in cardiac parameters among elite rowers and subelite rowers. Med Sci Sports Exerc. 2010;42:1215-20.

15. Weiner RB, Wang F, Isaacs SK, Malhotra R, Berkstresser B, Kim JH, Hutter AM, Jr., Picard MH, Wang TJ and Baggish AL. Blood pressure and left ventricular hypertrophy during American-style football participation. Circulation. 2013;128:524-31. 
16. Oxborough D, Sharma S, Shave R, Whyte G, Birch K, Artis N, Batterham AM and George $\mathrm{K}$. The right ventricle of the endurance athlete: the relationship between morphology and deformation. J Am Soc Echocardiogr. 2012;25:263-71.

17. D'Andrea A, Riegler L, Golia E, Cocchia R, Scarafile R, Salerno G, Pezzullo E, Nunziata L, Citro R, Cuomo S, Caso P, Di Salvo G, Cittadini A, Russo MG, Calabro R and Bossone E. Range of right heart measurements in top-level athletes: the training impact. International journal of cardiology. 2013;164:48-57.

18. La Gerche A, Burns AT, D'Hooge J, Macisaac AI, Heidbuchel H and Prior DL. Exercise Strain Rate Imaging Demonstrates Normal Right Ventricular Contractile Reserve and Clarifies Ambiguous Resting Measures in Endurance Athletes. J Am Soc Echocardiogr. 2011. 19. D'Andrea A, Riegler L, Morra S, Scarafile R, Salerno G, Cocchia R, Golia E, Martone F, Di Salvo G, Limongelli G, Pacileo G, Bossone E, Calabro R and Russo MG. Right ventricular morphology and function in top-level athletes: a three-dimensional echocardiographic study. Journal of the American Society of Echocardiography : official publication of the American Society of Echocardiography. 2012;25:1268-76.

20. Spence AL, Naylor LH, Carter HH, Buck CL, Dembo L, Murray CP, Watson P, Oxborough D, George KP and Green DJ. A prospective randomised longitudinal MRI study of left ventricular adaptation to endurance and resistance exercise training in humans. The Journal of physiology. 2011;589:5443-52.

21. Weiner RB, Hutter AM, Jr., Wang F, Kim J, Weyman AE, Wood MJ, Picard MH and Baggish AL. The impact of endurance exercise training on left ventricular torsion. JACC Cardiovasc Imaging. 2010;3:1001-9.

22. Abergel E, Chatellier G, Hagege AA, Oblak A, Linhart A, Ducardonnet A and Menard J. Serial left ventricular adaptations in world-class professional cyclists: implications for disease screening and follow-up. Journal of the American College of Cardiology. 2004;44:1449.

23. Maron BJ, Pelliccia A, Spataro A and Granata M. Reduction in left ventricular wall thickness after deconditioning in highly trained Olympic athletes. British heart journal. 1993;69:125-8.

24. Pelliccia A, Maron BJ, De Luca R, Di Paolo FM, Spataro A and Culasso F. Remodeling of left ventricular hypertrophy in elite athletes after long-term deconditioning. Circulation. 2002;105:944-9.

25. Weiner RB, Wang F, Berkstresser B, Kim J, Wang TJ, Lewis GD, Hutter AM, Jr., Picard MH and Baggish AL. Regression of "gray zone" exercise-induced concentric left ventricular hypertrophy during prescribed detraining. Journal of the American College of Cardiology. 2012;59:1992-4.

26. Corrado D, Pelliccia A, Heidbuchel H, Sharma S, Link M, Basso C, Biffi A, Buja G, Delise P, Gussac I, Anastasakis A, Borjesson M, Bjornstad HH, Carre F, Deligiannis A, Dugmore D, Fagard R, Hoogsteen J, Mellwig KP, Panhuyzen-Goedkoop N, Solberg E, Vanhees L, Drezner J, Estes NA, 3rd, Iliceto S, Maron BJ, Peidro R, Schwartz PJ, Stein R, Thiene G, Zeppilli P and McKenna WJ. Recommendations for interpretation of 12-lead electrocardiogram in the athlete. Eur Heart J. 2010;31:243-59.

27. Drezner JA, Fischbach P, Froelicher V, Marek J, Pelliccia A, Prutkin JM, Schmied CM, Sharma S, Wilson MG, Ackerman MJ, Anderson J, Ashley E, Asplund CA, Baggish AL, Borjesson M, Cannon BC, Corrado D, DiFiori JP, Harmon KG, Heidbuchel H, Owens DS, Paul S, Salerno JC, Stein R and Vetter VL. Normal electrocardiographic findings: recognising physiological adaptations in athletes. British journal of sports medicine. 2013;47:125-36. 
28. Sheikh N, Papadakis M, Ghani S, Zaidi A, Gati S, Adami PE, Carre F, Schnell F, Wilson M, Avila P, McKenna W and Sharma S. Comparison of electrocardiographic criteria for the detection of cardiac abnormalities in elite black and white athletes. Circulation. 2014;129:1637-49.

29. Dixon EM, Kamath MV, McCartney N and Fallen EL. Neural regulation of heart rate variability in endurance athletes and sedentary controls. Cardiovasc Res. 1992;26:713-9. 30. Huston TP, Puffer JC and Rodney WM. The athletic heart syndrome. $N$ Engl J Med. 1985;313:24-32.

31. Basavarajaiah S, Wilson M, Whyte G, Shah A, Behr E and Sharma S. Prevalence and significance of an isolated long QT interval in elite athletes. Eur Heart J. 2007;28:2944-9.

32. Rautaharju PM, Mason JW and Akiyama T. New age- and sex-specific criteria for QT prolongation based on rate correction formulas that minimize bias at the upper normal limits. International journal of cardiology. 2014;174:535-40.

33. Noseworthy PA, Weiner R, Kim J, Keelara V, Wang F, Berkstresser B, Wood MJ, Wang TJ, Picard MH, Hutter AM, Jr., Newton-Cheh C and Baggish AL. Early repolarization pattern in competitive athletes: clinical correlates and the effects of exercise training. Circ Arrhythm Electrophysiol. 2011;4:432-40.

34. Bailey JC, Watanabe AM, Besch HR, Jr. and Lathrop DA. Acetylcholine antagonism of the electrophysiological effects of isoproterenol on canine cardiac Purkinje fibers. Circ Res. 1979;44:378-83.

35. Barbosa EC, Bomfim Ade S, Benchimol-Barbosa PR and Ginefra P. Ionic mechanisms and vectorial model of early repolarization pattern in the surface electrocardiogram of the athlete. Ann Noninvasive Electrocardiol. 2008;13:301-7.

36. Pope HG, Jr., Kanayama G, Athey A, Ryan E, Hudson JI and Baggish A. The lifetime prevalence of anabolic-androgenic steroid use and dependence in Americans: current best estimates. Am J Addict. 2014;23:371-7.

37. Nottin S, Nguyen LD, Terbah M and Obert P. Cardiovascular effects of androgenic anabolic steroids in male bodybuilders determined by tissue Doppler imaging. The American journal of cardiology. 2006;97:912-5.

38. D'Andrea A, Caso P, Salerno G, Scarafile R, De Corato G, Mita C, Di Salvo G, Severino S, Cuomo S, Liccardo B, Esposito N and Calabro R. Left ventricular early myocardial dysfunction after chronic misuse of anabolic androgenic steroids: a Doppler myocardial and strain imaging analysis. British journal of sports medicine. 2007;41:149-55.

39. Baggish AL, Weiner RB, Kanayama G, Hudson JI, Picard MH, Hutter AM, Jr. and Pope $\mathrm{HG}$, Jr. Long-term anabolic-androgenic steroid use is associated with left ventricular dysfunction. Circ Heart Fail. 2010;3:472-6.

40. La Gerche A, Baggish AL, Knuuti J, Prior DL, Sharma S, Heidbuchel H and Thompson PD. Cardiac imaging and stress testing asymptomatic athletes to identify those at risk of sudden cardiac death. JACC Cardiovascular imaging. 2013;6:993-1007.

41. Maron BJ and Zipes DP. Introduction: eligibility recommendations for competitive athletes with cardiovascular abnormalities-general considerations. Journal of the American College of Cardiology. 2005;45:1318-21.

42. Haskell WL, Lee IM, Pate RR, Powell KE, Blair SN, Franklin BA, Macera CA, Heath GW, Thompson PD and Bauman A. Physical activity and public health: updated recommendation for adults from the American College of Sports Medicine and the American Heart Association. Circulation. 2007;116:1081-93. 


\section{Figure 1.}

Cardiac adaptation to physical training in athletes is a cycle dependent on the type of training, the duration of training and the intensity. Changes can be observed in both the electrocardiogram (top) and imaging of the myocardium (bottom). Isotonic stress, such as bicycling (the green pathway) leads to volume load and chamber dilation. Isometric stress, such as weightlifting (the yellow pathway) leads to pressure load and chamber hypertrophy. Rest quickly reverses the training changes seen in both cardiac structure and the electrocardiogram. 


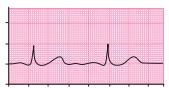

Normal
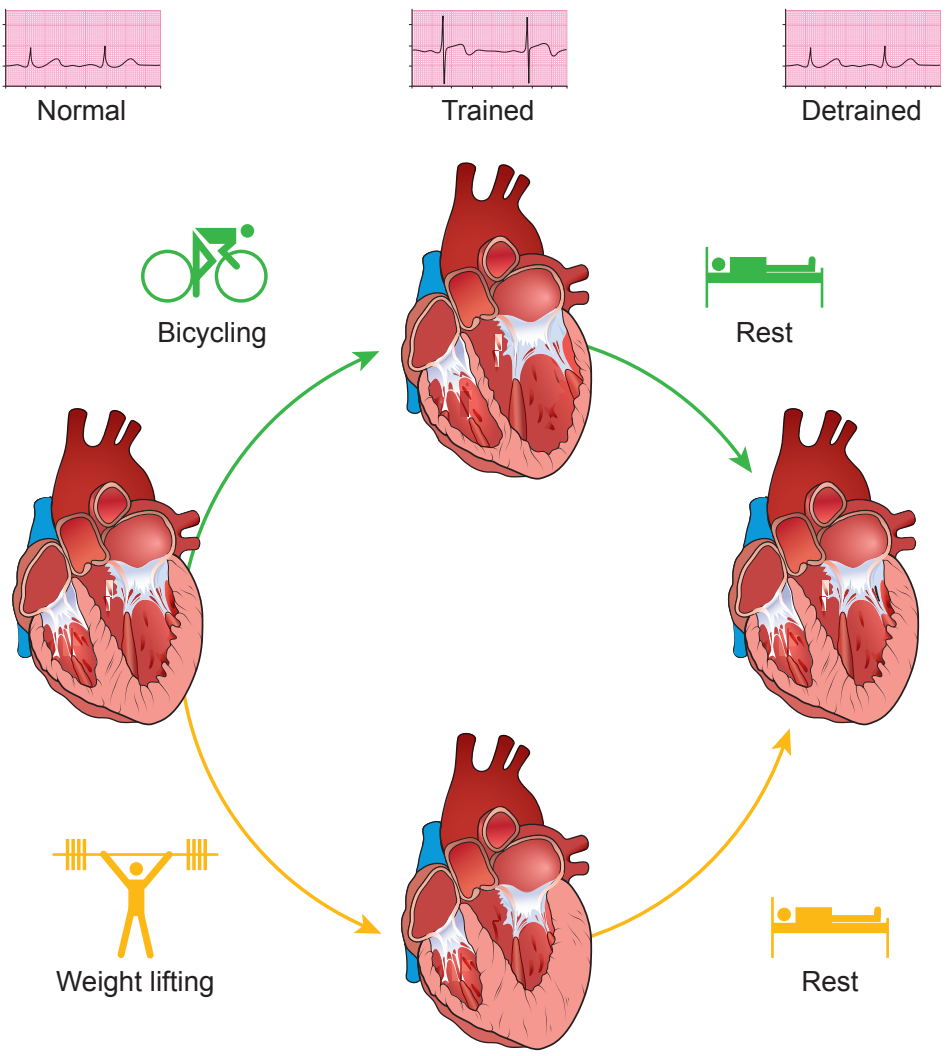